\title{
Retalho médio-frontal tunelizado para reconstrução nasal e em canto de olho em único tempo: uma série de casos
}

\section{Tunneled subcutaneous forehead flap in nasal and eye canthus defects by single-stage reconstruction: a serie of cases}

\author{
Bruno Mendes Segundo․ Ádilla Thaysa Mendes Ribeiro². Marco Augusto Sobreira Rocha Filhoํ․ Luis \\ Alberto Albano Ferreira ${ }^{3}$. \\ 1 Residente de Cirurgia de Cabeça e Pescoço, Hospital Universitário Walter Cantídio (HUWC), Fortaleza, Ceará, Brasil. \\ 2 Acadêmica de Medicina, Universidade Federal do Ceará (UFC), Fortaleza, Ceará, Brasil. 3 Chefe do Serviço de Cirurgia de \\ Cabeça e Pescoço, Hospital Universitário Walter Cantídio (HUWC), Fortaleza, Ceará, Brasil.
}

\section{RESUMO}

Introdução: a importância da face para o convívio social faz com que defeitos cirúrgicos na região representem desafios reconstrutivos. Particularmente as regiões do nariz e canto de olho apresentam dificuldades reconstrutivas inerentes ao tipo de pele e funções. Bastante utilizado, o retalho médio-frontal tem a desvantagem de necessitar de dois tempos operatórios, elevando gastos e expondo o paciente a semanas de condição estética bastante desagradável. Proposta por Kersidag, a modificação da técnica com a ressecção da pele do pedículo e tunelização subcutânea para região distante como a ponta de nariz permite a cirurgia em tempo único. Objetivo: o presente trabalho objetiva demonstrar que a técnica de Kersidag é segura e adequada. Metodologia: como procedimento metodológico, recorremos a referências bibliográficas sobre a temática e realizamos procedimento com base nas modificações descritas. Resultados: três pacientes do sexo feminino, de 64 a 81 anos foram submetidos à técnica, com reconstrução com excelente resultado estético e sem nenhuma perda de retalho em todos os casos. Conclusão: concluímos que a técnica de Kersidag é uma opção viável e segura para reconstrução de defeitos em ponta nasal e em canto de olho em único tempo sem prejuízos estéticos ou funcionais.

Palavras-chave: Procedimentos cirúrgicos reconstrutivos. Neoplasias cutâneas. Neoplasias de cabeça e pescoço.

\section{ABSTRACT}

Introduction: The importance of the face to the social living makes surgical defects in the region represent reconstructive challenges. In particular, the nose and eye corner regions present inherent reconstructive difficulties to the skin type and functions. Quite used, the mid-front flap has the disadvantage of needing two operative times, raising expenses and exposing the patient to weeks of a rather unpleasant aesthetic condition. Proposed by Kersidag, modification of the technique with the resection of the skin of the pedicle and subcutaneous tunneling to distant region as the nose tip allows surgery in single time. Objective: This study aims to demonstrate that the Kersidag technique is safe and adequate. Methodology: As a methodological procedure, we used bibliographical references on the subject and performed a procedure based on the modifications described. Results: Three female patients, aged 64 to 81 years, underwent the technique, with reconstruction with excellent aesthetic result and without loss of flap in all cases. Conclusion: We conclude that the Kersidag technique is a viable and safe option for reconstruction of nasal tip and eye corner defects in a single time without any aesthetic or functional damages.

Keywords: Reconstructive surgical procedures. Skin neoplasms. Head and neck neoplasms.

Autor correspondente: Bruno Mendes Segundo, Rua Julio Cesar, 1044, Bom Futuro, Fortaleza, Ceará. CEP: 60425-236. Telefone: +55 85 99746-1407. E-mail: brunosegundo@hotmail.com

Conflito de interesses: Não há qualquer conflito de interesses por parte de qualquer um dos autores.

Recebido em: 29 Ago 2017; Revisado em: 24 Set 2017; Aceito em: 28 Set 2017. 


\section{INTRODUÇÃO}

Há aproximadamente 600 anos a.C, o médico indiano Sushruta, em sua clássica obra Sushruta Samhita descreveu um método de reconstrução nasal com pele da fronte e pedículo vascularizado com detalhes suficientes para galgar-lhe o título de "pai da cirurgia plástica".

$\mathrm{Na}$ clássica obra de Res medica, Aulus Cornelius Celsus (53 a.C-7 d.C) escreveu seu nome na história da cirurgia reconstrutora com seus trabalhos sobre enxertos onde estudou e descreveu diversos defeitos de narizes, orelhas e lábios e suas reconstruções com retalhos de pele adjacente. ${ }^{1}$

Na renascença, quando as deformidades por sífilis e hanseníase eram comuns e afligiam milhares, o médico Gaspare Tagliacozzi desenvolveu, em 1597, um retalho autonomizado da base anterior do terço superior da face interna com pele do braço para reconstrução nasal total ou parcial. A técnica, usada amplamente por muitos anos é hoje conhecida como "técnica italiana". ${ }^{1-4}$

Apesar dos bons resultados, o aspecto socialmente pouco aceito de ter um braço aderido a face durante semanas contribuiu para o descrédito e escárnio do método italiano e das reconstruções nasais com retalhos por séculos. ${ }^{1}$

A reintrodução da técnica de Sushruta no ocidente se deu pela publicação de Lucas em 1794, na Gentlemen's Magazine, popularizando o termo de "retalho indiano".,

Os resultados eram considerados bons, mas as retrações da ponta do retalho e as infecções da área cruenta exposta na área doadora e na pele da "ponte" ainda eram complicações que falavam contra o método e levavam a críticas.

Os avanços para resolução de tais problemas se deram pelos estudos de Von Graefe, Carpue, Blandin e Dieffenbach que propuseram a dobradura da extremidade dos retalhos frontais a fim de reduzir a área cruenta. ${ }^{2,3}$ As dobras em ponta também proporcionaram uma reconstrução mais estética através do molde de columela e asas nasais.

O século XX trouxe avanços com a incorporação de enxertos condro-cutâneos ao retalho médio-frontal pediculado, permitindo ganhos funcionais e estéticos. ${ }^{1,3}$

Entretanto, a necessidade de dois tempos na reconstrução, às complicações como infecção, sangramento e retração que a "ponte" de pele proporcionava associado ao resultado estético pouco harmonioso no pós-operatório imediato ainda representavam um entrave no uso do retalho médio frontal.

A resolução de boa parte desses problemas se deu por meio da proposta inovadora de Kersidag, em 2008, com a primeira descrição de retalho médio-frontal com reconstrução em único tempo através da passagem do pedículo vascular sem pele por túnel de pele saudável. ${ }^{5}$

A técnica de Kersidag, apesar dos ótimos resultados, tem sido pouco repetida por alegações de que o retalho supostamente não resistiria à compressão do pedículo pela pele do túnel.

Nossa casuística de três casos objetiva demonstrar que a técnica é segura, factível e esteticamente adequada para reconstrução nasal e em canto de olho em um único tempo.

\section{MÉTODO}

A técnica empregada foi a modificação do retalho médio-frontal descrita por Kersidag e repetida com sucesso por Ebrahime e colaboradores. ${ }^{5,6}$

Três pacientes escolhidos pelos pesquisadores foram reconstruídos pela técnica descrita.

Todos os pacientes previamente assentiram e assinaram o termo de consentimento livre e esclarecido e permitiram a divulgação de suas imagens para fins de publicação científica.

\section{RELATO}

\section{Paciente 1}

Paciente do sexo feminino, 64 anos, com história de lesão em ponta nasal ressecada, cujo histopatológico evidenciou carcinoma basocelular tipo infiltrativo ulcerado ocupando até o subcutâneo com margens cirúrgicas da biópsia atingidas pela neoplasia. A síntese do defeito havia se dado por fechamento primário, ocasionando retração.

Após ressecção cirúrgica visando ampliação de margem da lesão, deu-se início à etapa de reconstrução da área ressecada.

Fez-se o desenho de uma ilha de pele na região médio-frontal e, em seguida, a secção do músculo frontal apenas superior e lateralmente, com preservação da gálea aponeurótica. $\mathrm{Na}$ região inferior da demarcação, próximo ao pedículo, seccionou-se apenas a pele, com a intenção de preservar a vascularização. Continuou-se a dissecação no plano submuscular até o pedículo arterial dominante, composto pelas artérias supraorbitária direita e supratroclear direita, ramos da artéria oftálmica, por sua vez um ramo da artéria carótida interna (Figura 1). Acompanham nervos de mesmo nome através dos supercílios e da fronte e emergem da caixa craniana por meio de forames de mesmo nome. Distribuem-se irrigando a musculatura e a pele da fronte e do couro cabeludo e a conjuntiva superior.

A seguir, confeccionou-se um túnel de pele subcutâneo comunicando a área doadora ao defeito cirúrgico, que serviu de passagem para o retalho até a região da ponta nasal, onde foi ancorado (Figura 2). Por fim, no mesmo tempo cirúrgico, fez-se o fechamento primário da área doadora. Trans e pósoperatório transcorreram sem intercorrências e a reconstrução mostrou-se estética e funcionalmente satisfatória (Figura 2).

Após 4 e 12 meses da cirurgia ficam ainda mais claras as vantagens desse tipo de retalho, evidenciando-se uma 
boa cicatrização, com a minimização de danos estéticos e preservação das linhas de expressão originais. Não houve retração, infecção ou necrose de retalho (Figuras 3 e 4).

\section{Paciente 2}

Paciente do sexo feminino, 68 anos, com recidiva de carcinoma espinocelular em conjuntiva bulbar, tarsal e globo-ocular esquerdo. Após a exenteração da órbita esquerda, incluindo a pálpebra superior, deu-se início à etapa de reconstrução da área ressecada usando técnica semelhante (Figura 5).

\section{Paciente 3}

Paciente do sexo feminino, 81 anos, com lesão em médio dorso nasal. A histopatologia revelou carcinoma basocelular tipo nodular. Procedeu-se à exérese e reconstrução usando a técnica descrita (Figura 6).

Com três semanas houve sofrimento da borda do retalho, sem necessidade de desbridamento cirúrgico. A cicatrização em três meses já apresentava resultado próximo ao final (Figura 7).

Figura 1. Marcação e confecção do retalho.
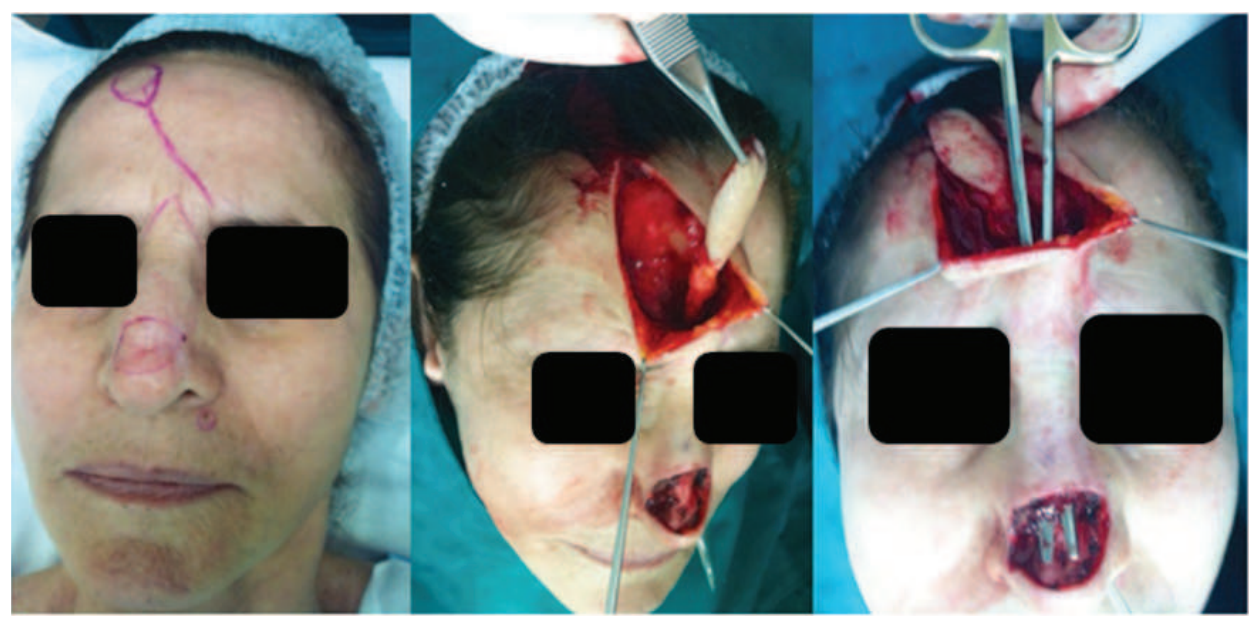

Figura 2. Pós-operatório imediato.

Figura 3. Pós-operatório 1 e 4 meses, respectivamente.

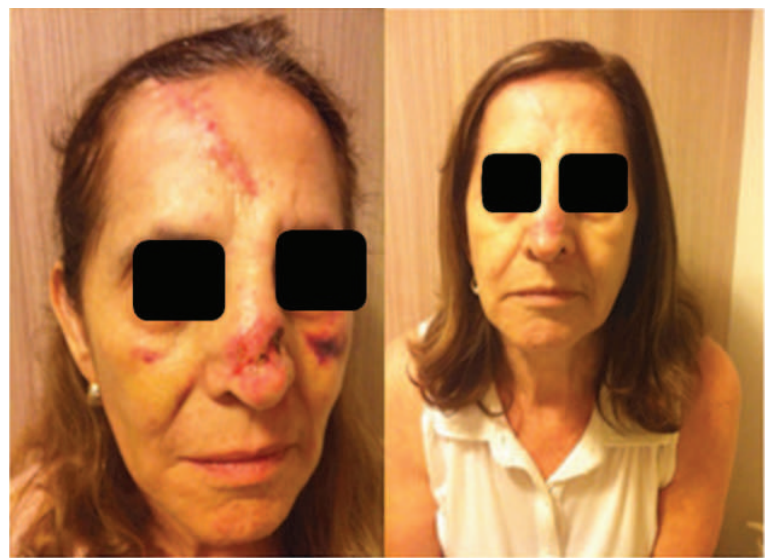

Figura 4. Pós-operatório 12 meses.

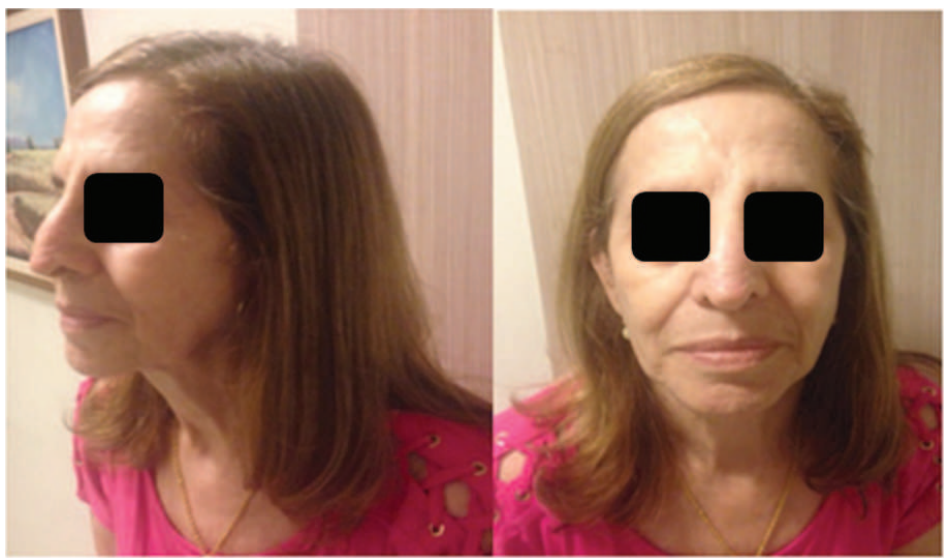

Figura 5. Pós-operatório imediato, com 1 semana e com 6 meses, respectivamente.

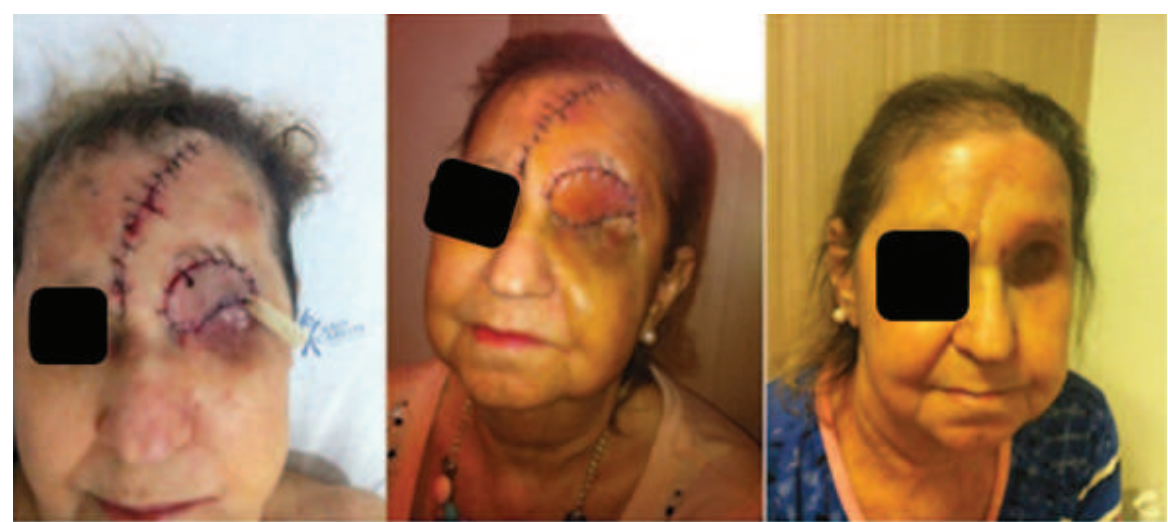


Figura 6. Intraoperatório e pós-operatório imediato.

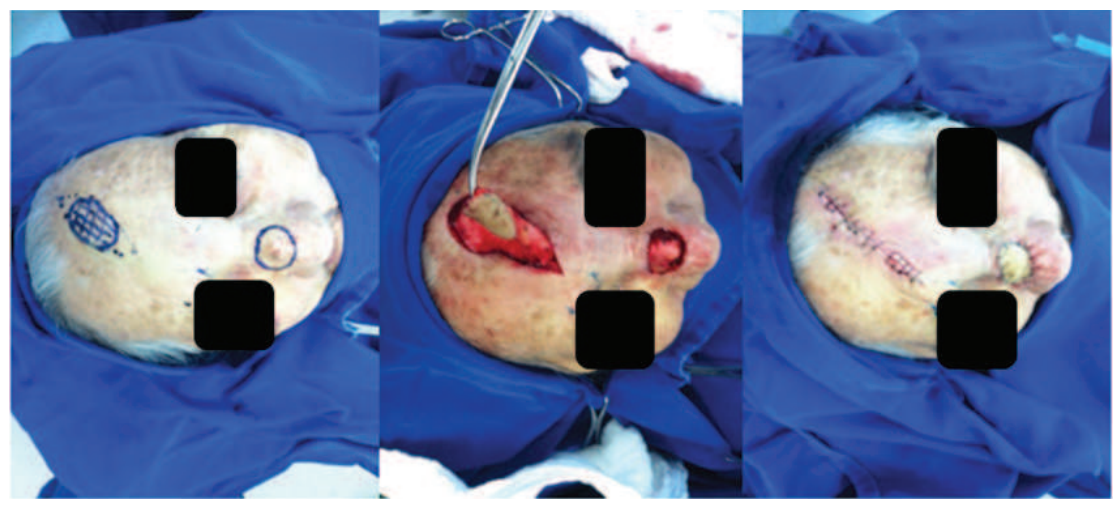

Figura 7. Pós-operatório com 3 semanas e com 3 meses, respectivamente.

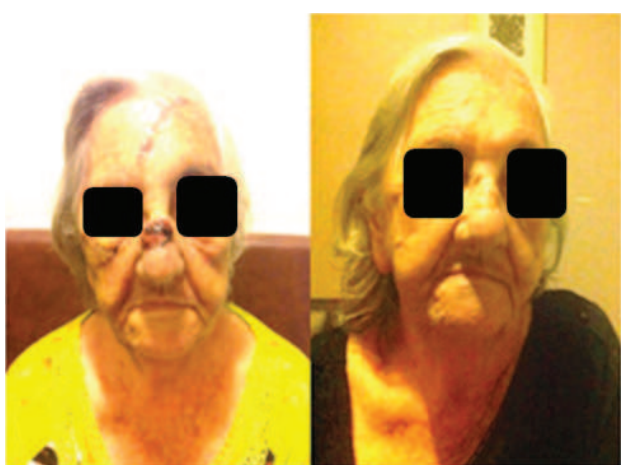

\section{DISCUSSÃO}

Em virtude da pele de espessura semelhante, proximidade com área receptora, duplo e calibroso pedículo arterial nutrício e relativa facilidade no aprendizado da técnica, o retalho médio-frontal clássico (retalho indiano) tem sido amplamente usado e aceito para reconstruções nasais, de canto de olho e palpebrais. $., 7,8,9$

A causa do defeito a ser corrigido varia amplamente de acordo com a casuística de cada centro, incluindo desde lesões benignas e traumas até ressecções por câncer.

A reconstrução nasal reparadora devido a tumores de pele é o mais relatado uso deste retalho, visto que as opções de reconstrução nasal, principalmente de ponta de nariz, não apresentarem resultados estéticos tão satisfatórios. ${ }^{3,10-12}$

Em nossa casuística, todas as pacientes foram submetidas à cirurgia de reconstrução após ressecção tumoral. A idade média foi de 71 anos e as pacientes eram todas do sexo feminino. As cirurgias foram realizadas em tempo único e não foram registradas distorções pós-operatórias, infecções, necrose de retalho ou formação de queloides.

Enquanto a literatura é vasta e antiga no uso do retalho médio-frontal em dois tempos, a experiência relatada da tunelização é mais restrita. Diversos autores propuseram a técnica para reconstruções em canto de olho, onde a rotação em torno de $90^{\circ}$ não seria um problema.

Já a tunelização do retalho para ponta nasal é pouco encontrada na literatura. Supostamente os receios de que um túnel longo e uma rotação de $180^{\circ}$ levariam a inviabilidade do retalho por compressão da pele adjacente ou torção do pedículo pareciam inibir tais tentativas, mas não apresenta respaldo na literatura ou em nossa casuística.

\section{REFERÊNCIAS}

1. Converse JM. Reconstructive plastic surgery. Philadelphia: Saunders; 1977. Volume 2, Corrective and reconstructive surgery of the nose; p. 858-1039.

2. Talmant JC. [Nose reconstruction. Parcial and total reconstruction].
Em 2008, Kersidag e colaboradores apresentaram a primeira série de lesões nasais corrigidas em um único tempo por um retalho médio-frontal tunelizado. Em sua casuística de 10 casos foram confeccionados retalhos de até $4 \mathrm{~cm} \times 2,5 \mathrm{~cm}$, seguidos por até 14 meses sem nenhuma deiscência, infecção, contratura ou perda de retalho. ${ }^{5}$

Ebrahime e colaboradores em série de oito casos publicada em 2010 realizaram reconstruções nasais com até $7 \mathrm{~cm} \times 5 \mathrm{~cm}$ sem nenhuma perda de retalho. ${ }^{6}$

A presente série é, até nosso conhecimento, a terceira série de casos, primeira no ocidente, de retalhos médio-frontais tunelizados para cobertura de defeitos nasais.

Todos os estudos convergem com a mesma conclusão de que a técnica é segura do ponto de vista de viabilidade do retalho e apresenta avanço significativo em uma técnica já consagrada, somando-se ainda a conveniência e economia de uma cirurgia realizada em único tempo.

A vantagem de ressocialização precoce do paciente, pela inexistência de deformidade transitória causada pela ponte de pele é uma vantagem adicional de extrema relevância.

\section{CONCLUSÃO}

O retalho médio-frontal ilhado tunelizado apresentou um resultado estético e funcional satisfatório, sendo bastante seguro do ponto de vista vascular e trazendo inovações que asseguram vantagens aos pacientes e cirurgiões. É uma experiência de importância indiscutível, que serve de base para que a reconstrução em regiões da face tenha um salto qualitativo e, desse modo, possa atender melhor às necessidades dos pacientes.

Techniques chirurgicales: chirurgie plastique reconstructive et esthétique. Elsevier; 2000. French.

3. Rohrich RJ, Barton FE, Hollier L. Nasal reconstruction. In: Aston SJ, Beasley RW, Thorne CHM, editors. Grabb and Smith's plastic 
surgery. New York: Lippincott-Raven; 1997. p. 513-29.

4. Champaneria MC, Workman AD, Gupta SC. Sushruta: father of plastic surgery. Ann Plast Surg. 2014;73(1):2-7.

5. Karsidag S, Sacak B, Bayraktaroglu S, Ozcan A, Ugurlu K, Bas L. A novel approach for the construction of medial canthal and nasal dorsal defects: frontal hairline island lap. J Craniofacial Surg. 2008;19(6): 1653-7.

6. Ebrahimil A, Motamedi MH, Nejadsarvari N, Koushki ES. Subcutaneous forehead island flap for nasal reconstruction. Iran Red Crescent Med J. 2012;14(5):271-5.

7. Cintra HP, Bouchama A, Holanda T, Jaimovich CA, Pitanguy I. Uso do retalho médio-frontal na reconstrução do nariz. Rev Bras Cir Plástica. 2013;28(2):212-7.

8. Menick FJ. A 10-year experience in nasal reconstruction with the three-stage forehead flap. Plast Reconst Surg. 2002;109(6):1839-55.
9. Menick FJ. Aesthetic refinements in use of forehead for nasal reconstruction: the paramedian forehead flap. Clin Plast Surg. 1990;17(4):607-22.

10. Anbar RA, Arruda AM, Reis GC, Santos L, Anbar RA. Reconstrução nasal com emprego de retalho médio-frontal. Rev Bras Cir Plást. 2010;25(1):208-10.

11. Lima BS, Abdalla SC, Vasconcellos ZA, Vieira VJ, Bins-Ely J, Neves R. Reconstrução nasal com retalho frontal: nossa experiência. Arq Catarin Med. 2007;36:103-5.

12. André MC, Fraga A, Garcia CR, Pignatelli JG, Soares RO. Retalho em ilha de tubarão: uma técnica cirúrgica reconstrutiva de defeitos localizados na área nasal alar/perialar. Um procedimento simples. An Bras Dermatol. 2011;86(4 Supl 1):160-3.

\section{Como citar:}

Mendes B Segundo, Ribeiro AT, Rocha MA Filho, Ferreiro LA. Retalho médio-frontal tunelizado para reconstrução nasal e em canto de olho em único tempo: uma série de casos. Rev Med UFC. 2017 jan-mar;58(1):75-79. 